\title{
Interior Tomography Using 1D Generalized Total Variation. Part I: Mathematical Foundation*
}

\author{
John Paul Ward ${ }^{\dagger}$, Minji Lee ${ }^{\ddagger}$, Jong Chul Ye , and Michael Unser ${ }^{\dagger}$
}

\begin{abstract}
Motivated by the interior tomography problem, we propose a method for exact reconstruction of a region of interest of a function from its local Radon transform in any number of dimensions. Our aim is to verify the feasibility of a one-dimensional reconstruction procedure that can provide the foundation for an efficient algorithm. For a broad class of functions, including piecewise polynomials and generalized splines, we prove that an exact reconstruction is possible by minimizing a generalized total variation seminorm along lines. The main difference with previous works is that our approach is inherently one-dimensional and that it imposes less constraints on the class of admissible signals. Within this formulation, we derive unique reconstruction results using properties of the Hilbert transform, and we present numerical examples of the reconstruction.
\end{abstract}

Key words. interior tomography, perfect reconstruction, generalized total variation

AMS subject classifications. $34 \mathrm{M} 50,44 \mathrm{~A} 12,44 \mathrm{~A} 15$

DOI. $10.1137 / 140982428$

1. Introduction. We consider the general problem of recovering a function on a region of interest $\Omega_{\mu} \subset \mathbb{R}^{d}$ using information from the local Radon transform. In the two-dimensional setting, this corresponds to the interior tomography problem, where one assumes that line integrals passing through the region of interest are known, while the others are unknown [17]. Our reconstruction method is based on the identities

$$
\begin{aligned}
& I=(-1)^{(d-1) / 2}(2 \pi)^{1-d} B_{\varphi_{0}} \frac{\partial^{d-1}}{\partial s^{d-1}} \mathcal{R} I, \\
& I=(-1)^{d / 2+1}(2 \pi)^{1-d} \mathcal{H}_{\varphi_{0}} B_{\varphi_{0}} \frac{\partial^{d-1}}{\partial s^{d-1}} \mathcal{R} I
\end{aligned}
$$

for odd- and even-dimensional spaces, respectively. They provide a method for inverting the Radon transform $\mathcal{R} I$ of an image $I$, using a modified backprojection operator $B_{\varphi_{0}}$ and the directional Hilbert transform $\mathcal{H}_{\varphi_{0}}$; cf. $[15,23]$. In the even-dimensional case, this is

\footnotetext{
* Received by the editors August 14, 2014; accepted for publication (in revised form) November 25, 2014; published electronically January 22, 2015. The research of the authors was funded by the European Research Council under the European Union's Seventh Framework Programme (FP7/2007-2013) / ERC grant agreement 267439. This work was also funded in part by the Swiss National Science Foundation under grant 200020-144355. Preliminary results of this work appeared in [12].

http://www.siam.org/journals/siims/8-1/98242.html

${ }^{\dagger}$ Biomedical Imaging Group, Ecole Polytechnique Fédérale de Lausanne (EPFL), CH-1015, Lausanne, Switzerland (john.ward@epfl.ch, michael.unser@epfl.ch).

${ }^{\ddagger}$ Bio Imaging and Signal Processing Lab, Department of Bio and Brain Engineering, KAIST, Korea (minjilee@ kaist.ac.kr, jong.ye@kaist.ac.kr). The research of these authors was supported by Basic Science Research Program through the National Research Foundation of Korea (NRF) funded by Ministry of Science, ICT, and Future Planning (NRF-2009-0081089).
} 
different from the standard filtered backprojection, since the Hilbert transform follows the backprojection step. An advantage of our approach is that we can segment the domain into lines and reconstruct on these restricted domains. Consequently, we reduce the reconstruction to a one-dimensional problem, which simplifies the formulation.

We consider images modeled as piecewise smooth functions. In particular, the function should be defined on a partitioned domain such that it is smooth on each subregion. Previous works addressed this problem under the assumption that the function was a piecewise polynomial $[18,20]$, and the authors concentrated on the piecewise linear images [18, Theorem 4], due to the complexity of their two-dimensional regularization term.

By combining a simplified one-dimensional formulation with the ideas developed in $[18,20]$, we show how to perfectly reconstruct new classes of functions in the ideal, noise-free setting. For example, any function that has the form of a nonuniform exponential spline along lines in the plane fits our framework, and the nonpolynomial versions were not previously considered. More generally, the function to be reconstructed should be a generalized L-spline along a set of lines that pass through the region of interest.

We also provide results for the case where the linear restrictions are combinations of polynomial splines of various orders. This is a direct extension of $[18,20]$, since every piecewise polynomial function satisfies our condition.

This paper is organized as follows. In the remainder of section 1, we introduce our notation and provide details on our reconstruction method. Section 2 contains the fundamental results of the paper. We fully explain the odd-dimensional case and focus on the more complicated even-dimensional case. For $d$ even, we show how to perfectly reconstruct functions that are generalized L-splines along lines. In section 3, we formulate the multispline reconstruction method, and in section 4 we present numerical experiments.

1.1. Notation. The variable $\boldsymbol{\theta}$ denotes a vector on the unit sphere $\mathbb{S}^{d-1} \subset \mathbb{R}^{d}$. The collection of vectors that are orthogonal to $\boldsymbol{\theta}$ is denoted as

$$
\boldsymbol{\theta}^{\perp}=\left\{\boldsymbol{y} \in \mathbb{R}^{d}: \boldsymbol{y} \cdot \boldsymbol{\theta}=0\right\} .
$$

The variables $\varphi$ and $\phi$ are used similarly.

Since our motivation is tomography, we refer to real-valued functions in the spatial domain as images and denote them as $I(\boldsymbol{x})$ for $\boldsymbol{x} \in \mathbb{R}^{d}$. While $I$ is defined on the whole space, we assume that it is supported on the unit ball; i.e., $I$ is supported within

$$
\Omega_{1}:=\left\{\boldsymbol{x}=\rho \boldsymbol{\theta} \in \mathbb{R}^{d}:|\rho| \leq 1, \boldsymbol{\theta} \in \mathbb{S}^{d-1}\right\} .
$$

The interior of $I$ is its restriction to the ball $\Omega_{\mu}=\left\{\rho \boldsymbol{\theta} \in \mathbb{R}^{d}:|\rho|<\mu\right\}$ for some $\mu<1$, which we assume to be fixed throughout the paper.

We denote the Radon transform of an image $I$ as

$$
\mathcal{R} I(\boldsymbol{\theta}, s)=\int_{\boldsymbol{\theta}^{\perp}} I(s \boldsymbol{\theta}+\boldsymbol{y}) \mathrm{d} \boldsymbol{y},
$$

where $s \in \mathbb{R}$ and $\boldsymbol{\theta} \in \mathbb{S}^{d-1}$, and we use the term sinogram for any function in the Radon domain, regardless of the number of dimensions $d$. The local Radon transform of $I$ is the restriction of $\mathcal{R} I$ to the region $\{(\boldsymbol{\theta}, s):|s|<\mu\}$. 
In this paper, we consider a regularization term that is defined by a Fourier multiplier operator L. For example, we can choose L to be a constant coefficient differential operator

$$
a_{K} \mathrm{D}^{K}+a_{K-1} \mathrm{D}^{K-1}+\cdots+a_{1} \mathrm{D}+a_{0},
$$

where $K \geq 1$ and each $a_{k}$ is a complex number; here, D denotes the distributional derivative on $\mathbb{R}$. Importantly, for any open interval $E \subset \mathbb{R}$, such operators map $C_{c}^{\infty}(E)$ to $C_{c}(E)$, and their null spaces are composed of entire functions. In the example above, the null space is finite-dimensional, and it comprises products of polynomials and complex exponentials.

On an open interval $E \subset \mathbb{R}$, an operator L defines a seminorm

$$
\|f\|_{T V(\mathrm{~L} ; E)}:=\|\mathrm{L} f\|_{L_{1}(E)}
$$

for sufficiently smooth functions $f$. We extend this definition using the dual formulation

$$
\|f\|_{T V(\mathrm{~L} ; E)}:=\sup \left\{\int_{E} f(x) \mathrm{L}^{*} h(x) \mathrm{d} x: h \in C_{c}^{\infty}(E),\|h\|_{\mathrm{L}_{\infty}} \leq 1\right\}
$$

to allow for a broader class of functions.

The Fourier transform of a function $f \in L_{1}\left(\mathbb{R}^{d}\right)$ is

$$
\widehat{f}(\boldsymbol{\omega})=\mathcal{F}\{f\}(\boldsymbol{\omega})=\int_{\mathbb{R}^{d}} f(\boldsymbol{x}) e^{-2 \pi i \boldsymbol{x} \cdot \boldsymbol{\omega}} \mathrm{d} \boldsymbol{x},
$$

and if $\widehat{f} \in L_{1}\left(\mathbb{R}^{d}\right)$, its inverse Fourier transform is

$$
\mathcal{F}^{-1}\{f\}(\boldsymbol{x})=\int_{\mathbb{R}^{d}} \widehat{f}(\boldsymbol{\omega}) e^{2 \pi i \boldsymbol{x} \cdot \boldsymbol{\omega}} \mathrm{d} \boldsymbol{\omega} .
$$

The Hilbert transform $[9,15]$ of a one-dimensional function $f: \mathbb{R} \rightarrow \mathbb{R}$ is

$$
\begin{aligned}
\mathcal{H} f(x) & =\frac{1}{\pi} P V \int_{\mathbb{R}} \frac{f(x-y)}{y} \mathrm{~d} y \\
& =\frac{1}{\pi} \lim _{\epsilon \rightarrow 0} \int_{|y|>\epsilon} \frac{f(x-y)}{y} \mathrm{~d} y, \\
\mathcal{F}\{\mathcal{H} f\}(\omega) & =-i \operatorname{sgn}(\omega) \widehat{f}(\omega),
\end{aligned}
$$

whenever the right-hand side is well defined. For a given vector $\varphi_{0} \in \mathbb{S}^{d-1} \subset \mathbb{R}^{d}$, the directional Hilbert transform $[10,15]$ of a $d$-dimensional function $f: \mathbb{R}^{d} \rightarrow \mathbb{R}$ is

$$
\begin{aligned}
\mathcal{H}_{\varphi_{0}} f(\boldsymbol{x}) & =\frac{1}{\pi} P V \int_{\mathbb{R}} \frac{f\left(\boldsymbol{x}-s \boldsymbol{\varphi}_{0}\right)}{s} \mathrm{~d} s, \\
\mathcal{F}\left\{\mathcal{H}_{\boldsymbol{\varphi}_{0}} f\right\}(\boldsymbol{\omega}) & =-i \operatorname{sgn}\left(\boldsymbol{\omega} \cdot \boldsymbol{\varphi}_{0}\right) \widehat{f}(\boldsymbol{\omega})
\end{aligned}
$$

whenever the right-hand side is well defined. 
1.2. Reconstruction method. For the reconstruction of an image $I$ from its Radon transform $\mathcal{R} I$, we use the differentiated backprojection $[7,15,17,19,23]$. The reconstruction formulas are given in the introduction, where the backprojection operator

$$
B_{\boldsymbol{\varphi}_{0}} J(\boldsymbol{x})=\int_{\mathbb{S}^{d-1} \cap\left\{\phi \cdot \varphi_{0} \geq 0\right\}} J(\boldsymbol{\phi}, \boldsymbol{x} \cdot \boldsymbol{\phi}) \mathrm{d} \boldsymbol{\phi}
$$

depends on the vector $\varphi_{0}$.

In the following, we analyze the continuity properties of the involved operators and provide sufficient conditions for a function to be recovered using this method. To make this precise, we require additional notation. For the domain $\mathbb{S}^{d-1} \times \mathbb{R}$ and $\alpha \geq 0$, we define the Sobolev space $H^{\alpha}\left(\mathbb{S}^{d-1} \times \mathbb{R}\right)$ by the equation

$$
\|J\|_{H^{\alpha}\left(\mathbb{S}^{d-1} \times \mathbb{R}\right)}^{2}=\int_{\mathbb{S}^{d-1}} \int_{-\infty}^{\infty}\left(1+\sigma^{2}\right)^{\alpha}|\mathcal{F}\{J(\boldsymbol{\varphi}, \cdot)\}(\sigma)|^{2} \mathrm{~d} \sigma \mathrm{d} \boldsymbol{\varphi} .
$$

Similarly, the Sobolev spaces $H^{\alpha}\left(\mathbb{R}^{d}\right)$ are defined by

$$
\|I\|_{H^{\alpha}\left(\mathbb{R}^{d}\right)}^{2}=\int_{\mathbb{R}^{d}}\left(1+|\boldsymbol{\omega}|^{2}\right)^{\alpha}|\widehat{I}(\boldsymbol{\omega})|^{2} \mathrm{~d} \boldsymbol{\omega} .
$$

The Schwartz space of infinitely differentiable, rapidly decaying functions on $\mathbb{R}^{d}$ is denoted as $\mathcal{S}\left(\mathbb{R}^{d}\right)$.

Theorem 1.1 (see [13, p. 42]). For each $\alpha \geq 0$, there exist constants $c(\alpha, d), C(\alpha, d)$ such that for any $I \in \mathcal{S}\left(\mathbb{R}^{d}\right)$ that is supported on the closed unit ball,

$$
c(\alpha, d)\|I\|_{H^{\alpha}\left(\mathbb{R}^{d}\right)} \leq\|\mathcal{R} I\|_{H^{\alpha+(d-1) / 2}\left(\mathbb{S}^{d-1} \times \mathbb{R}\right)} \leq C(\alpha, d)\|I\|_{H^{\alpha}\left(\mathbb{R}^{d}\right)} .
$$

The Schwartz space on $\mathbb{S}^{d-1} \times \mathbb{R}$ is denoted as $\mathcal{S}\left(\mathbb{S}^{d-1} \times \mathbb{R}\right)$ (cf. [13, p. 10]), which can be defined as restrictions of functions from $\mathcal{S}\left(\mathbb{R}^{d+1}\right)$.

Definition 1.2. Let $X$ be the seminormed space of tempered distributions

$$
\left\{g(\boldsymbol{\varphi}, s)=\frac{\partial^{d-1}}{\partial s^{d-1}} \mathcal{R} I(\varphi, s): I \in L_{\infty}\left(\mathbb{R}^{d}\right), \operatorname{supp}(I) \subseteq \Omega_{1}\right\}
$$

with

$$
|g|_{X}:=\|\mathcal{R} I\|_{H^{(d-1) / 2}\left(\mathbb{S}^{d-1} \times \mathbb{R}\right)} .
$$

Lemma 1.3. For $J \in \mathcal{S}\left(\mathbb{S}^{d-1} \times \mathbb{R}\right)$, the operator $B_{\varphi_{0}}$ is defined in the Fourier domain by

$$
\mathcal{F}\left\{B_{\varphi_{0}} J\right\}(\boldsymbol{\omega})=|\boldsymbol{\omega}|^{1-d} \widehat{J}\left(\operatorname{sgn}\left(\boldsymbol{\omega} \cdot \boldsymbol{\varphi}_{0}\right) \frac{\boldsymbol{\omega}}{|\boldsymbol{\omega}|}, \operatorname{sgn}\left(\boldsymbol{\omega} \cdot \boldsymbol{\varphi}_{0}\right)|\boldsymbol{\omega}|\right) .
$$

Proof. This formula can be verified using the techniques described in [13, pp. 13-15]. Details are provided in Appendix A.

In the previous lemma, we see how the backprojection operator depends on the domain of integration in (1.2). If we had instead used the standard backprojection

$$
\widetilde{B} J(\boldsymbol{x})=\int_{\mathbb{S}^{d-1}} J(\boldsymbol{\phi}, \boldsymbol{x} \cdot \boldsymbol{\phi}) \mathrm{d} \boldsymbol{\phi}
$$

Copyright (C) by SIAM. Unauthorized reproduction of this article is prohibited. 
that integrates over the sphere $\mathbb{S}^{d-1}$, then the Fourier transform would be

$$
\mathcal{F}\{\widetilde{B} J\}(\boldsymbol{\omega})=|\boldsymbol{\omega}|^{1-d}\left(\widehat{J}\left(\frac{\boldsymbol{\omega}}{|\boldsymbol{\omega}|},|\boldsymbol{\omega}|\right)+\widehat{J}\left(-\frac{\boldsymbol{\omega}}{|\boldsymbol{\omega}|},-|\boldsymbol{\omega}|\right)\right) .
$$

Lemma 1.4. For $I \in \mathcal{S}\left(\mathbb{R}^{d}\right)$, the following reconstruction formulas are valid:

$$
\begin{aligned}
& I=(-1)^{(d-1) / 2}(2 \pi)^{1-d} B_{\varphi_{0}} \frac{\partial^{d-1}}{\partial s^{d-1}} \mathcal{R} I, \\
& I=(-1)^{d / 2+1}(2 \pi)^{1-d} \mathcal{H}_{\varphi_{0}} B_{\varphi_{0}} \frac{\partial^{d-1}}{\partial s^{d-1}} \mathcal{R} I .
\end{aligned}
$$

Proof. The Fourier slice theorem implies that $\mathcal{R} I \in \mathcal{S}\left(\mathbb{S}^{d-1} \times \mathbb{R}\right)$, and it follows that $g(\boldsymbol{\theta}, s):=\frac{\partial^{d-1}}{\partial s^{d-1}} \mathcal{R} I(\boldsymbol{\theta}, s)$ is also in this Schwartz space. Then Lemma 1.3 implies that

$$
\begin{aligned}
\mathcal{F}\left\{B_{\boldsymbol{\varphi}_{0}} g\right\}(\boldsymbol{\omega}) & =|\boldsymbol{\omega}|^{1-d} \widehat{g}\left(\operatorname{sgn}\left(\boldsymbol{\omega} \cdot \boldsymbol{\varphi}_{0}\right) \frac{\boldsymbol{\omega}}{|\boldsymbol{\omega}|}, \operatorname{sgn}\left(\boldsymbol{\omega} \cdot \boldsymbol{\varphi}_{0}\right)|\boldsymbol{\omega}|\right) \\
& =|\boldsymbol{\omega}|^{1-d}\left(\left(2 \pi i \operatorname{sgn}\left(\boldsymbol{\omega} \cdot \boldsymbol{\varphi}_{0}\right)|\boldsymbol{\omega}|\right)^{d-1} \mathcal{F}\{\mathcal{R} I\}\left(\operatorname{sgn}\left(\boldsymbol{\omega} \cdot \boldsymbol{\varphi}_{0}\right) \frac{\boldsymbol{\omega}}{|\boldsymbol{\omega}|}, \operatorname{sgn}\left(\boldsymbol{\omega} \cdot \boldsymbol{\varphi}_{0}\right)|\boldsymbol{\omega}|\right)\right) \\
& =(2 \pi i)^{d-1}\left(\operatorname{sgn}\left(\boldsymbol{\omega} \cdot \boldsymbol{\varphi}_{0}\right)\right)^{d-1} \widehat{I}(\boldsymbol{\omega}) .
\end{aligned}
$$

If $d$ is odd, i.e., $d=2 k_{o}+1$ for some integer $k_{o} \geq 1$, then

$$
\mathcal{F}\left\{B_{\varphi_{0}} g\right\}(\boldsymbol{\omega})=(-1)^{k_{o}}(2 \pi)^{2 k_{o}} \widehat{I}(\boldsymbol{\omega})
$$

and

$$
\begin{aligned}
B_{\varphi_{0}} \frac{\partial^{d-1}}{\partial s^{d-1}} \mathcal{R} I & =B_{\varphi_{0}} g \\
& =(-1)^{k_{o}}(2 \pi)^{2 k_{o}} I .
\end{aligned}
$$

Now, if $d$ is even, i.e., $d=2 k_{e}$ for some integer $k_{e} \geq 1$, then

$$
\mathcal{F}\left\{B_{\boldsymbol{\varphi}_{0}} g\right\}(\boldsymbol{\omega})=(-1)^{k_{e}}(2 \pi)^{2 k_{e}-1}(-i) \operatorname{sgn}\left(\boldsymbol{\omega} \cdot \boldsymbol{\varphi}_{0}\right) \widehat{I}(\boldsymbol{\omega})
$$

and

$$
\begin{aligned}
B_{\varphi_{0}} \frac{\partial^{d-1}}{\partial s^{d-1}} \mathcal{R} I & =B_{\varphi_{0}} g \\
& =(-1)^{k_{e}}(2 \pi)^{2 k_{e}-1} \mathcal{H}_{\varphi_{0}} I
\end{aligned}
$$

In general, the restriction $I \in \mathcal{S}$ is too strong. Below, we provide a continuity result for the proposed backprojection operator that we combine with the Sobolev estimates of Theorem 1.1 to obtain weaker conditions for reconstruction.

Lemma 1.5. The operator $B_{\varphi_{0}}: X \rightarrow L_{2}\left(\mathbb{R}^{d}\right)$ is continuous.

Proof. Let $g(\boldsymbol{\theta}, s)=\frac{\partial^{d-1}}{\partial s^{d-1}} \mathcal{R} I(\boldsymbol{\theta}, s)$ be an element of $X \bigcap \mathcal{S}\left(\mathbb{S}^{d-1} \times \mathbb{R}\right)$. 
If $d$ is odd, then there are constants $C, \widetilde{C}>0$ such that

$$
\begin{aligned}
\left\|B_{\varphi_{0}} g\right\|_{L_{2}\left(\mathbb{R}^{d}\right)} & =C\|I\|_{L_{2}\left(\mathbb{R}^{d}\right)} \\
& \leq \widetilde{C}\|\mathcal{R} I\|_{H^{(d-1) / 2}\left(\mathbb{S}^{d-1} \times \mathbb{R}\right)} .
\end{aligned}
$$

The inequality follows from Theorem 1.1.

In the case where $d$ is even, we use the fact that the directional Hilbert transform is unitary. Then we apply a technique similar to the odd-dimensional case to obtain the result.

In the proof of the previous result, we have made use of the density of Schwartz class functions in $X$. The operator $B_{\varphi_{0}}$ is defined on $X$ using this property. More generally, $B_{\varphi_{0}}$ can be defined on all tempered distributions using the fact that it is the adjoint of the Radon operator; cf. Proposition A.1.

Theorem 1.6. The reconstruction formulas of Lemma 1.4 are valid for images $I \in L_{\infty}\left(\mathbb{R}^{d}\right)$ with support in $\Omega_{1}$. Furthermore, the seminorm on the space $X$ of Definition 1.2 is in fact a norm, so $X$ is a normed space.

Proof. The assumptions imply that each such $I$ is a compactly supported element of $L_{2}\left(\mathbb{R}^{d}\right)$. Therefore, the first part of the theorem is valid due to Theorem 1.1 and Lemma 1.5.

The fact that $X$ is a normed space is an immediate consequence of the validity of the reconstruction formulas on $X$.

2. Single spline perfect reconstruction from local data. Our purpose is to use regularization to exactly reconstruct the interior of an image $I$ from its local Radon transform. In spaces of odd dimension, reconstruction is straightforward. The derivative is a local operator, and for $|\boldsymbol{x}|<\mu, B_{\boldsymbol{\varphi}_{0}} J(\boldsymbol{x})$ only depends on the value of $J(\boldsymbol{\phi}, s)$ for $|s| \leq|\boldsymbol{x}|<\mu$. Therefore, we have an explicit formula (1.4) for the reconstruction of the region of interest; cf. [13, pp. 20-21].

Proposition 2.1. If $d$ is odd and $I \in L_{\infty}\left(\mathbb{R}^{d}\right)$ is supported on $\Omega_{1}$, then $I$ can be recovered from $\mathcal{R} I$ using local operations.

Proof. The fact that $B_{\varphi_{0}}$ is a local operator on elements of $X$ can be deduced using the fact that it is the adjoint of the Radon operator; cf. Proposition A.1.

In spaces of even dimension, the Hilbert transform introduces difficulties, and it is this setting that we consider for the remainder of the paper. In what follows, we assume that $d$ is even.

Since the directional Hilbert transform is equivalent to one-dimensional Hilbert transforms along lines, we formulate our reconstruction to take advantage of this and to progressively reconstruct images along lines.

We use $l_{0}=\left(\rho_{0}, \boldsymbol{\theta}_{0}, \boldsymbol{\varphi}_{0}\right)$ to represent the line $\rho_{0} \boldsymbol{\theta}_{0}+\tau \boldsymbol{\varphi}_{0}$, where $\boldsymbol{\theta}_{0} \cdot \boldsymbol{\varphi}_{0}=0$. We also use the notation

$$
l_{0} \bigcap \Omega_{\mu}=\left\{\tau \in \mathbb{R}:\left|\rho_{0} \boldsymbol{\theta}_{0}+\tau \boldsymbol{\varphi}_{0}\right|<\mu\right\} .
$$

Definition 2.2. Let $\mathcal{I}_{\mathrm{L}}$ be the collection of real-valued images $I \in L_{2}\left(\mathbb{R}^{d}\right)$ satisfying the following conditions:

(i) there is a set of lines $\mathcal{L}$ such that the ball of radius $\mu$ centered at the origin is contained in the union of the elements of $\mathcal{L}$;

(ii) $I \in L_{\infty}\left(\mathbb{R}^{d}\right)$ is supported on $\Omega_{1}$;

Copyright $\odot$ by SIAM. Unauthorized reproduction of this article is prohibited. 


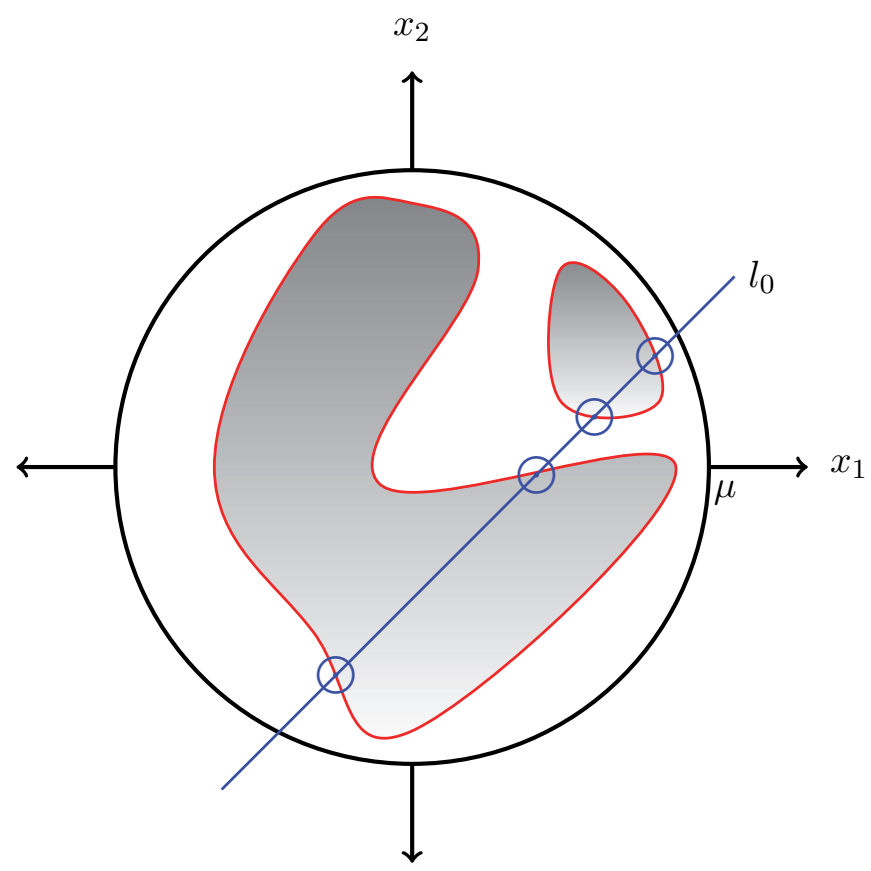

Figure 1. Structure diagram: This diagram illustrates the reconstruction. We assume the line $l_{0}$ is contained in $\mathcal{L}$, so that the restriction of $I$ to $l_{0}$ is an $\mathrm{L}$-spline. The knots of the nonuniform spline are depicted by the symbol $\odot$. The shaded regions denote structural elements within $I$, and we expect spline knots to appear at the boundaries.

(iii) for every $l_{0}=\left(\rho_{0}, \boldsymbol{\theta}_{0}, \boldsymbol{\varphi}_{0}\right) \in \mathcal{L}$, there are $N_{l_{0}} \in \mathbb{Z}_{\geq 0}$ real coefficients $\left\{a_{n}^{l_{0}}\right\}_{n=1}^{N_{\theta_{0}}}$ and points $\left\{\tau_{n}^{l_{0}}\right\}_{n=1}^{N_{l_{0}}}$ such that

$$
\mathrm{L} I\left(\rho_{0} \boldsymbol{\theta}_{0}+\tau \boldsymbol{\varphi}_{0}\right)=\sum_{n=1}^{N_{l_{0}}} a_{n}^{l_{0}} \delta\left(\tau-\tau_{n}^{l_{0}}\right)
$$

within $\Omega_{\mu}$.

Essentially, this definition says that every element $I$ of $\mathcal{I}_{\mathrm{L}}$ should satisfy the following property: for every $l_{0} \in \mathcal{L}, I\left(\rho_{0} \boldsymbol{\theta}_{0}+\tau \boldsymbol{\varphi}_{0}\right)$ is a generalized L-spline. To be precise, a generalized $\mathrm{L}$-spline is a function that is composed of smooth pieces that are in the null space of $\mathrm{L}$, and these pieces are joined at points $\tau_{n}^{l_{0}}$ of finite smoothness called spline knots; cf. Figure 1.

The nature of the reconstruction reduces the problem of exact recovery to a one-dimensional problem. Therefore, our analysis focuses on showing that we have perfect reconstruction on lines from $\mathcal{L}$. Note, however, that this implies perfect reconstruction over the entire domain $\Omega_{\mu}$.

For exact recovery of an image $I$ on a line $l_{0}$, we minimize a generalized total variation (TV) seminorm. Since $\mathcal{R} I(\boldsymbol{\varphi}, s)$ is known for $s<\mu$, any ambiguity $g \in L_{\infty}(\mathbb{R})$ will have a Hilbert transform $\mathcal{H} g$ that is zero on $l_{0} \bigcap \Omega_{\mu}$.

Definition 2.3. For $I \in \mathcal{I}_{\mathrm{L}}$ and $l_{0} \in \mathcal{L}$, the set $\mathcal{G}$ consists of every compactly supported, real-valued function $g \in L_{\infty}(\mathbb{R})$ that satisfies the following: $\mathcal{H} g(\tau)=0$ for $\tau \in l_{0} \bigcap \Omega_{\mu}$. Also, 
define the set

$$
\mathcal{G}_{I, l_{0}}:=\left\{F(\tau)=I\left(\rho_{0} \boldsymbol{\theta}_{0}+\tau \boldsymbol{\varphi}_{0}\right)+g(\tau): g \in \mathcal{G}\right\} .
$$

Essentially, $\mathcal{G}_{I, l_{0}}$ is the set of functions that are compatible with the Hilbert data of $I$ on the line $l_{0}$.

Our main result on perfect reconstruction is stated below. The key point is that the elements of $\mathcal{G}$ are infinitely smooth on $l_{0} \cap \Omega_{\mu}$. This is in contrast with the image $I$ that is to be reconstructed; along lines, $I$ is a generalized L-spline, where $\mathrm{L}$ is a finite order operator. This gap in smoothness allows us to distinguish between the these two terms using regularization.

Theorem 2.4. Let $I \in \mathcal{I}_{\mathrm{L}}$, and fix $l_{0}$ so that

$$
\mathrm{L} I\left(\rho_{0} \boldsymbol{\theta}_{0}+\tau \boldsymbol{\varphi}_{0}\right)=\sum_{n=1}^{N_{l_{0}}} a_{n}^{l_{0}} \delta\left(\tau-\tau_{n}^{l_{0}}\right)
$$

on $\Omega_{\mu}$. Then

$$
\left.\underset{F \in \mathcal{G}_{I, l_{0}}}{\arg \min }\|F\|_{T V\left(\mathrm{~L} ; l_{0}\right.} \cap \Omega_{\mu}\right)
$$

is the one element set $\left\{I\left(\rho_{0} \boldsymbol{\theta}_{0}+\tau \boldsymbol{\varphi}_{0}\right)\right\}$.

Proof. Lemma 2.6 implies that any element $F_{0}$ of (2.2) is a function of the form $I\left(\rho_{0} \boldsymbol{\theta}_{0}+\right.$ $\left.\tau \boldsymbol{\varphi}_{0}\right)+g(\tau)$, where $g$ is equal to a function in the null space $\mathcal{N}_{\mathrm{L}}$ on $l_{0} \bigcap \Omega_{\mu}$. In addition, we know that the Radon transform of $g$ is equal to 0 for $|s|<\mu$, so Lemma 2.7 implies that $g$ is identically 0 .

This theorem can be viewed as an example of the general principle that splines minimize TV-type functionals over restricted classes of functions. In contrast to the classical setting [5], where interpolation conditions are imposed, we restrict the functions using local Hilbert data as in [18].

2.1. Technical lemmas. Here, we present the lemmas used in the proof of Theorem 2.4. Lemma 2.5 addresses the smoothness of the perturbations $g \in \mathcal{G}$. Lemma 2.6 restricts the class of possible minimizers of the generalized TV seminorm, and Lemma 2.7 demonstrates the uniqueness of the minimizer.

Lemma 2.5. If $g \in \mathcal{G}$, then $g$ has continuous derivatives of all orders on $l_{0} \cap \Omega_{\mu}$.

Proof. A formula for $g$ is

$$
\begin{aligned}
g(\tau) & =-\mathcal{H}_{\varphi_{0}} \mathcal{H}_{\varphi_{0}} g(\tau) \\
& =-\frac{1}{\pi} P V \int_{\mathbb{R}} \frac{\mathcal{H}_{\varphi_{0}} g(\tau-s)}{s} \mathrm{~d} s .
\end{aligned}
$$

Changing variables, we have

$$
\begin{aligned}
g(\tau) & =-\frac{1}{\pi} P V \int_{\mathbb{R}} \frac{\mathcal{H}_{\varphi_{0}} g(t)}{\tau-t} \mathrm{~d} t \\
& =-\frac{1}{\pi} P V \int_{\mathbb{R} \backslash\left(l_{0} \cap \Omega_{\mu}\right)} \frac{\mathcal{H}_{\varphi_{0}} g(t)}{\tau-t} \mathrm{~d} t .
\end{aligned}
$$

Copyright $\odot$ by SIAM. Unauthorized reproduction of this article is prohibited. 
Then the restriction $\tau \in l_{0} \bigcap \Omega_{\mu}$ means that the singularity is located outside of the domain of integration. Therefore $g$ has derivatives of all orders.

Lemma 2.6. Let $I \in \mathcal{I}_{\mathrm{L}}$, and fix $l_{0}$ so that for $\tau \in l_{0} \bigcap \Omega_{\mu}$

$$
\mathrm{L} I\left(\rho_{0} \boldsymbol{\theta}_{0}+\tau \boldsymbol{\varphi}_{0}\right)=\sum_{n=1}^{N_{l_{0}}} a_{n}^{l_{0}} \delta\left(\tau-\tau_{n}^{l_{0}}\right) .
$$

Then

$$
\underset{F \in \mathcal{G}_{I, l_{0}}}{\arg \min }\|F\|_{T V\left(\mathrm{~L} ; l_{0} \cap \Omega_{\mu}\right)}
$$

is contained in

$$
\left\{I\left(\rho_{0} \boldsymbol{\theta}_{0}+\tau \boldsymbol{\varphi}_{0}\right)+g(\tau):\left.g(\tau)\right|_{l_{0} \cap \Omega_{\mu}}=\left.P\right|_{l_{0} \cap \Omega_{\mu}}, P \in \mathcal{N}_{\mathrm{L}}\right\}
$$

Proof. Suppose that

$$
F(\tau)=I\left(\rho_{0} \boldsymbol{\theta}_{0}+\tau \boldsymbol{\varphi}_{0}\right)+g(\tau) \in \mathcal{G}_{I, l_{0}}
$$

is not in (2.3). Then Lemma 2.5 implies that there is an interval $\left[e_{1}, e_{2}\right] \subset l_{0} \bigcap \Omega_{\mu}$, not containing any point $\tau_{n}^{l_{0}}$, where $\mathrm{L} g(\tau)>0($ or $\mathrm{L} g(\tau)<0)$. Then

$$
\begin{aligned}
\|F\|_{T V\left(\mathrm{~L} ; l_{0} \cap \Omega_{\mu}\right)} & =\sup _{\|h\|_{L_{\infty}} \leq 1} \int_{l_{0} \cap \Omega_{\mu}} F(t) \mathrm{L}^{*} h(t) \mathrm{d} t \\
& =\sup _{\|h\|_{L_{\infty}} \leq 1} \int_{l_{0} \cap \Omega_{\mu}}\left(I\left(\rho_{0} \boldsymbol{\theta}_{0}+t \boldsymbol{\varphi}_{0}\right)+g(t)\right) \mathrm{L}^{*} h(t) \mathrm{d} t \\
& =\sup _{\|h\|_{L_{\infty}} \leq 1} \sum_{n=1}^{N_{l_{0}}} a_{n}^{l_{0}} h\left(\tau_{n}^{l_{0}}\right)+\int_{l_{0} \cap \Omega_{\mu}} \mathrm{L} g(t) h(t) \mathrm{d} t .
\end{aligned}
$$

Note that in the formulation above, the functions $h$ are assumed to be smooth test functions as in (1.1). Now define a sequence of smooth functions $\left\{h_{m}\right\}_{m=1}^{\infty}$ converging to a function $h_{0}$ satisfying

(i) $h_{0}\left(\tau_{n}^{\theta_{0}}\right)=\operatorname{sgn}\left(a_{n}^{l_{0}}\right)$;

(ii) $h_{0}(t)=1$ for $t \in\left[e_{1}, e_{2}\right]$;

(iii) $h_{0}(t)=0$ for every other value of $t$.

Considering this sequence, we know that

$$
\begin{aligned}
\|F\|_{T V\left(\mathrm{~L} ; l_{0} \cap \Omega_{\mu}\right)} & \geq \sum_{n=1}^{N_{l_{0}}}\left|a_{n}^{l_{0}}\right|+\int_{e_{1}}^{e_{2}}|\mathrm{~L} g(t)| \mathrm{d} t \\
& >\sum_{n=1}^{N_{l_{0}}}\left|a_{n}^{l_{0}}\right| \\
& =\left\|I\left(\rho_{0} \boldsymbol{\theta}_{0}+\tau \boldsymbol{\varphi}_{0}\right)\right\|_{T V\left(\mathrm{~L} ; l_{0} \cap \Omega_{\mu}\right)} .
\end{aligned}
$$

Copyright (C) by SIAM. Unauthorized reproduction of this article is prohibited. 
In the following lemma, we use properties of the Hilbert transform to demonstrate the uniqueness of the minimizer of the TV seminorm. This result is a generalization of Lemma 2 from [18], in which the authors proved the result for polynomial functions. Here, we see that essentially the same statement holds for entire functions in general.

Lemma 2.7. Let $v \in L_{2}(\mathbb{R})$, and denote its Hilbert transform as $w:=\mathcal{H} v$. Let $P: \mathbb{C} \rightarrow \mathbb{C}$ be an entire function taking real values on the real line, and suppose that $w(x)=P(x)$ on the real line segment $-e_{0} \leq x \leq e_{0}$ for some $e_{0}>0$. If $v(x)=0$ for $-e_{0} \leq x \leq e_{0}$, then $v$ and $w$ are both identically 0.

Proof. The proof relies on the characterization of Hilbert transform pairs as boundary values of analytic functions on the upper half of the complex plane [8, section 4.1.2], [9, section 3.2] and on the Schwarz reflection principle [2, p. 125].

We define the domains

$$
\begin{aligned}
& D_{1}:=\{z=x+i y \in \mathbb{C}: y>0\} \bigcup\left\{z=x+i y \in \mathbb{C}:-e_{0}<x<e_{0}, y=0\right\}, \\
& D_{2}=\mathbb{C} \backslash\left\{z=x+i y \in \mathbb{C}:|x| \geq e_{0}, y=0\right\},
\end{aligned}
$$

and we define the function $F: D_{1} \rightarrow \mathbb{C}$ by

$$
\begin{aligned}
F(z) & :=\frac{1}{\pi} \int_{\mathbb{R}} \frac{v(s)}{z-s} \mathrm{~d} s \\
& =\frac{1}{\pi} \int_{\mathbb{R}} v(s) \frac{x-s}{(x-s)^{2}+y^{2}} \mathrm{~d} s-i \frac{1}{\pi} \int_{\mathbb{R}} v(s) \frac{y}{(x-s)^{2}+y^{2}} \mathrm{~d} s,
\end{aligned}
$$

where $z=x+i y$. The function $F$ is analytic on the upper half plane, and we can directly verify that it is continuous on $D_{1}$, since $v$ is equal to zero in a neighborhood of the origin.

On the segment $\left(-e_{0}, e_{0}\right), F(x)=w(x)$, which means that $F$ is real-valued on this interval. Therefore, the Schwarz reflection principle implies that $F$ can be continued analytically to all of $D_{2}$.

Since $F$ is equal to an entire function $P$ on an interval, $F=P$ on the domain $D_{2}$ [11, p. 90].

Now, notice that the kernel of the integral in the imaginary part of $F$ is the Poisson kernel. This implies that the imaginary part of $F$ converges to $-v$ as $y \rightarrow 0^{+}[16$, section 3.2]. The kernel in the real part of $F$ is the conjugate Poisson kernel, so the real part of $F$ converges to $w$ as $y \rightarrow 0^{+}[8$, section 4.1.2]. Therefore,

$$
\begin{aligned}
w(x)-i v(x) & =\lim _{y \rightarrow 0^{+}} F(x+i y) \\
& =\lim _{y \rightarrow 0^{+}} P(x+i y) \\
& =P(x)
\end{aligned}
$$

Since $P$ is real-valued on the real line, $w$ is equal to $P$ and $v$ is identically 0 . Since the Hilbert transform is unitary, $w$ is also identically 0 . 
3. Multispline models. In this section, we aim to extend our model and allow for images whose one-dimensional projections are linear combinations of splines of different orders.

Definition 3.1. Let $\mathcal{I}^{K}$ be the collection of images $I \in L_{2}\left(\mathbb{R}^{d}\right)$ satisfying the following:

(i) there is a set of lines $\mathcal{L}$ such that the ball of radius $\mu$ centered at the origin is contained in the union of the elements of $\mathcal{L}$;

(ii) $I \in L_{\infty}\left(\mathbb{R}^{d}\right)$ is supported on $\Omega_{1}$;

(iii) for every $l_{0} \in \mathcal{L}$, there are polynomial splines $\left\{I_{k}^{l_{0}}\right\}_{k=1}^{K}$, where $I_{k}^{l_{0}}$ is of order $k$ and

$$
I\left(\rho_{0} \boldsymbol{\theta}_{0}+\tau \boldsymbol{\varphi}_{0}\right)=\sum_{k=1}^{K} I_{k}^{l_{0}}(\tau) .
$$

Notice that $\mathcal{I}^{K}$ contains all images that are piecewise polynomials. Specifically, if there is a partition of the interior domain $\Omega_{\mu}$ into a finite collection of subdomains, on which $I$ is a polynomial, then $I \in \mathcal{I}^{K}$ for some value of $K$.

The idea for perfect reconstruction is similar to the single spline case. We seek to extract a finitely smooth spline from a signal that is perturbed by an infinitely smooth term. The added difficulty is the fact that we combine splines of different orders. As a result, we perform the recovery process in stages. We begin by reconstructing an arbitrary perturbation of the signal of interest. Then we extract the splines successively from the perturbed signal, starting with the lowest order first. After identifying the splines, there remains a polynomial ambiguity that is removed using the local Radon transform data.

Definition 3.2. Let $E=\left(e_{1}, e_{2}\right)$ be an open interval in $\mathbb{R}$. The set $\mathcal{G}^{K}(E)$ consists of every compactly supported, real-valued function $g \in L_{\infty}(\mathbb{R})$ that satisfies the following: $\mathcal{H} g(\tau)=0$ for $\tau \in E$ and $\mathrm{d}^{k} g / \mathrm{d} x^{k} \in L_{\infty}(E) \bigcap L_{1}(E)$ for $k \leq K+2$.

3.1. Perfect reconstruction from local data. The first step in reconstructing $I \in \mathcal{I}^{K}$ is to extend the local Radon data $\mathcal{R} I$ to a function of the form $\mathcal{R}(I+G)$ for some $G \in$ $L_{\infty}\left(\mathbb{R}^{d}\right)$ satisfying the following: $G$ is compactly supported, $\mathcal{R} G(\varphi, s)=0$ for $s<\mu$, and $G\left(\rho_{0} \boldsymbol{\theta}_{0}+\tau \boldsymbol{\varphi}_{0}\right) \in \mathcal{G}^{K}\left(l_{0} \cap \Omega_{\mu}\right)$. Then we apply the reconstruction formula of (1.5). On a fixed $l_{0} \in \mathcal{L}$, the resulting function is $I\left(\rho_{0} \boldsymbol{\theta}_{0}+\tau \boldsymbol{\varphi}_{0}\right)+g(\tau)$, where $g$ is the ambiguity term.

Note that $I\left(\rho_{0} \boldsymbol{\theta}_{0}+\tau \boldsymbol{\varphi}_{0}\right)+g(\tau)$ satisfies the conditions of Lemma 3.3 with $K_{0}=1$. We apply the regularization described in the lemma, and we identify the first order spline $I_{1}^{l_{0}}(\tau)$ up to an order 1 (degree 0 ) polynomial ambiguity $q_{1}(\tau)$; i.e., the regularization produces $I_{1}^{l_{0}}(\tau)+q_{1}(\tau)$. Then

$$
I\left(\rho_{0} \boldsymbol{\theta}_{0}+\tau \boldsymbol{\varphi}_{0}\right)+g(\tau)-\left(I_{1}^{l_{0}}+q_{1}\right)(\tau)
$$

satisfies the conditions of Lemma 3.3 with $K_{0}=2$. We repeat the regularization until all spline terms of $I\left(\rho_{0} \boldsymbol{\theta}_{0}+\tau \boldsymbol{\varphi}_{0}\right)$ have been identified, up to a polynomial ambiguity; i.e., we repeat the regularization until we have identified

$$
q(\tau)+I\left(\rho_{0} \boldsymbol{\theta}_{0}+\tau \boldsymbol{\varphi}_{0}\right)=q(\tau)+\sum_{k=1}^{K} I_{k}^{l_{0}}(\tau),
$$

where $q$ is a polynomial of order $K$.

Copyright @ ( by SIAM. Unauthorized reproduction of this article is prohibited. 
In the spatial domain, we have now identified two functions:

1. the initial reconstruction $I\left(\rho_{0} \boldsymbol{\theta}_{0}+\tau \boldsymbol{\varphi}_{0}\right)+g(\tau)$ (defined on $\left.\mathbb{R}\right)$;

2. the sum of splines and polynomials $q(\tau)+\sum_{k=1}^{K} I_{k}^{l_{0}}(\tau)$ (defined on $l_{0} \bigcap \Omega_{\mu}$ ), obtained by regularization.

The difference between these two functions is $g-q$. We then apply the regularization of Lemma 3.4 to this function, and we obtain $g$. Subtracting $g$ from $I\left(\rho_{0} \boldsymbol{\theta}_{0}+\tau \boldsymbol{\varphi}_{0}\right)+g(\tau)$, we finish the process.

3.2. Technical lemmas. The basis of our multispline reconstruction process is the following lemma. It shows how to separate splines using regularization.

Lemma 3.3. Let $E=\left(e_{1}, e_{2}\right)$ be an open interval in $\mathbb{R}$. Suppose $f$ is a sum of order $k$ splines $f_{k}$ and a smooth function $g \in \mathcal{G}^{K}(E)$; i.e., $f=g+\sum_{k=K_{0}}^{K_{1}} f_{k}$. Then

$$
\underset{\left\|F_{1}\right\|_{T V\left(\mathrm{D}^{K_{0}+1}, E\right)}}{\arg \min }\left\|f-F_{1}\right\|_{T V\left(\mathrm{D}^{\left.K_{0}, E\right)}\right.}
$$

is equal to the set of functions

$$
\left\{f_{K_{0}}+q: q \in \mathcal{N}_{\mathrm{D}^{K_{0}}}\right\}
$$

Proof. This can be interpreted as an approximation result. We write $f=\left(f-F_{1}\right)+F_{1}$, and we must identify the best approximation to $\mathrm{D}^{K_{0}} f$ from all functions $\mathrm{D}^{K_{0}} F_{1}$ satisfying $\left\|F_{1}\right\|_{T V\left(\mathrm{D}^{K_{0}+1}, E\right)}<\infty$. This condition implies that $\mathrm{D}^{K_{0}} F_{1}$ is of bounded variation. Now, by definition,

$$
\mathrm{D}^{K_{0}} f=\mathrm{D}^{K_{0}} f_{K_{0}}+\mathrm{D}^{K_{0}}\left(g+\sum_{k=K_{0}+1}^{K_{1}} f_{k}\right) .
$$

The first term $\mathrm{D}^{K_{0}} f_{K_{0}}$ is a sum of Dirac deltas, while the second term is piecewise continuous. In this form, we see that the best approximation to $\mathrm{D}^{K_{0}} f$ from the class of bounded variation functions is the second term on the right-hand side of (3.1). Therefore $\mathrm{D}^{K_{0}}\left(f-F_{1}\right)=\mathrm{D}^{K_{0}} f_{K_{0}}$, and the only ambiguity comes from the null space of $\mathrm{D}^{K_{0}}$.

Lemma 3.4. Let $E=\left(e_{1}, e_{2}\right)$ be an open interval in $\mathbb{R}$, and suppose $q$ is a polynomial of order $K \in \mathbb{Z}_{\geq 1}$. If $g \in \mathcal{G}^{K}(E)$, then

$$
\{g\}=\underset{\widetilde{g} \in \mathcal{G}^{K}(E)}{\arg \min }\|g-q-\widetilde{g}\|_{T V\left(\mathrm{D}^{K} ; E\right)} .
$$

Proof. We first note that the solution set is contained in

$$
\left\{\widetilde{g} \in \mathcal{G}^{K}(E):\left.\widetilde{g}\right|_{E}=\left.g\right|_{E}+p, p \in \mathcal{N}_{\mathrm{D}^{K}}\right\},
$$

where every element has a Hilbert transform $\mathcal{H} \widetilde{g}(t)$ that is zero for $t \in E$. We apply Lemma 2.7 by defining $v:=\mathcal{H} \widetilde{g}$, and this implies that the null space term must be zero.

Copyright @ by SIAM. Unauthorized reproduction of this article is prohibited. 
4. Interior tomography application. Based on the differentiated backprojection (DBP) reconstruction in two- or three-dimensional space, the original reconstruction problem can be converted to a set of one-dimensional Hilbert transform problems [3, 4, 21]. If the DBP data $g$ is available for the support of the object $I$, the reconstruction can simply be done by performing a one-dimensional truncated inverse Hilbert transform. ${ }^{1}$ However, in the case of the interior tomography problem, the detector is truncated, so the DBP data is only available within a field of view (FOV). In this section, we assume that the FOV covers the region of interest (ROI). In fact, we assume that they are equivalent. For the exact reconstruction at a given point on a line $l_{0}$, it is sufficient to know the DBP data $g$ on the intersection between $l_{0}$ and the support of $I$. However, this condition is not satisfied in interior tomography problems.

The main problem with inverting a truncated Hilbert transform is the existence of a null space. More specifically, suppose we want to reconstruct a function $f$ on an interval $E=\left(e_{1}, e_{2}\right)$, and we only know $\mathcal{H} f$ on $E$. There exist nonzero functions $g$ such that

$$
\mathcal{H} \tilde{f}(x)=0, \quad x \in E .
$$

In fact, we can take any $g \in L_{2}(\mathbb{R})$ that is supported outside of $E$, and the following formula will produce such an $\widetilde{f}$ :

$$
-\frac{1}{\pi} \int_{\mathbb{R} \backslash E} \frac{g(t)}{t-\tau} \mathrm{d} \tau
$$

One approach to solving this problem is to use a regularized reconstruction, where the regularization term takes advantage of a priori knowledge of the function $f$ to specify the proper null space term.

4.1. Interior tomography with known subregions. In [3], the authors assume that the image $I$ to be reconstructed is known on small subregions within the FOV; cf. Figure 2. In this figure, the FOV is located completely inside of the object. Therefore, on $l_{0}$, the DBP is not known over the support of $I$, so $I$ cannot be recovered using the inverse Hilbert transform. However, if there are known subregions in $(-\mu, \mu)$ such as $\left(e_{1}, e_{2}\right)$ and $\left(e_{3}, e_{4}\right)$, the authors show that $I$ can be determined uniquely on this line.

The authors of [3] also note that the solution to the problem can be found using a sequence of projection operators. The optimized solution was found by projection onto convex sets (POCS). Let us denote $\left.I\right|_{l_{0}}$ as $\bar{f}$. Also, let us assume $g:=\mathcal{H} \bar{f}$ is known on $(-\mu, \mu)$. Then the five convex constraints are

$$
\begin{aligned}
& C_{1}:=\left\{f \in L_{2}(\mathbb{R}): f(t)=0,|t|>1\right\}, \\
& C_{2}:=\left\{f \in L_{2}(\mathbb{R}): \mathcal{H} f(t)=g(t),|t|<\mu\right\}, \\
& C_{3}:=\left\{f \in L_{2}(\mathbb{R}): f(t)=\bar{f}(t), t \in\left(e_{1}, e_{2}\right) \bigcup\left(e_{3}, e_{4}\right)\right\}, \\
& C_{4}:=\left\{f \in L_{2}(\mathbb{R}): \frac{1}{\pi} \int_{-1}^{1} f(t) \mathrm{d} t=C_{\bar{f}}\right\}, \\
& C_{5}:=\left\{f \in L_{2}(\mathbb{R}): f(t) \geq 0,|t|<1\right\} .
\end{aligned}
$$

\footnotetext{
${ }^{1}$ Note that $g$ and $I$ are related by the directional Hilbert transform; cf. (1.5).
} 


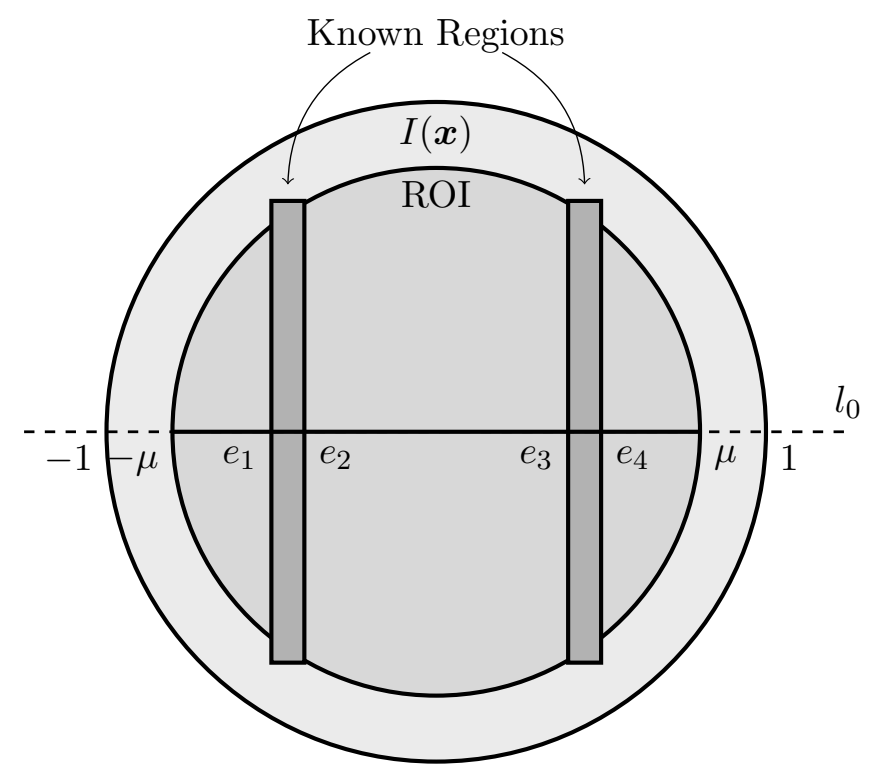

Figure 2. Structure diagram: Interior problem with known subregions.

If $P_{C_{j}}$ is the orthogonal projector onto the convex set $C_{j}$ for $j=1, \ldots, 5$, then the iteration of the POCS algorithm is described as

$$
f_{m}=P_{C_{5}} P_{C_{4}} P_{C_{3}} P_{C_{2}} P_{C_{1}} f_{m-1}
$$

with some initialization $f_{0}$. If the intersection of the constraint sets is not empty, this algorithm converges to one element of the intersection. The simulation results show that this method works well; however, in practice, it is difficult to know the intensity inside of the object.

4.2. Exact recovery under generalized TV penalty. For interior tomography without known subregions, we propose to use the generalized TV penalty in place of the projection onto the convex set $C_{3}$. Specifically, we consider the new set

$$
\widetilde{C}_{3}:=\left\{f \in L_{2}(\mathbb{R}):\|f\|_{T V\left(L: E_{\mu}\right)}<\gamma\right\},
$$

where $E_{\mu}:=l_{0} \bigcap \Omega_{\mu}$. This algorithm corresponds to the single operator reconstruction of Theorem 2.4.

We recall that in our model, we assume $\bar{f} \in L_{\infty}$. For the reconstruction algorithm, we further assume that we know upper and lower bounds $B_{u}, B_{l} \geq 0$ for $\bar{f}$, and we define

$$
C_{\bar{f}}:=\left\{f \in L_{2}(\mathbb{R}): B_{l} \leq f(t) \leq B_{u}\right\} .
$$

We propose to use the POCS algorithm (4.1) with $P_{C_{3}}$ replaced by $P_{\widetilde{C}_{3}} P_{C_{\bar{f}}} \cdot{ }^{2}$

\footnotetext{
${ }^{2}$ Note that $P_{C_{\bar{f}}}$ makes the last projection $P_{C_{5}}$ unnecessary, so we eliminate this step from our algorithm.
} 
The projection to each constraint is quite straightforward except for the projection on $\widetilde{C}_{3}$, which is implemented as a denoising step. For a given $f_{0} \in C_{\bar{f}}$, the projection on $\widetilde{C}_{3}$ is determined as

$$
P_{\widetilde{C}_{3}} f_{0}=\underset{f \in C_{\bar{f}}}{\arg \min }\left\{\left\|f-f_{0}\right\|_{L_{2}\left(E_{\mu}\right)}^{2}+2 \lambda\|f\|_{T V\left(\mathrm{~L} ; E_{\mu}\right)}\right\}
$$

for an appropriate Lagrange multiplier $\lambda$. Using the definition of the generalized TV seminorm, the problem is written as

$$
\min _{f \in C_{\bar{f}}} \sup _{h \in C_{c}^{\infty}\left(E_{\mu}\right),\|h\|_{L_{\infty}} \leq 1}\left\{\left\|f-f_{0}\right\|_{L_{2}\left(E_{\mu}\right)}^{2}+2 \lambda \int_{E_{\mu}} f(t) \mathrm{L}^{*} h(t) \mathrm{d} t\right\} .
$$

In most cases, we can weaken the restrictions on the functions $h$. If $\mathrm{L}$ is an operator of order at most $K$, i.e., $\mathrm{L}: C^{K}(\mathbb{R}) \rightarrow C(\mathbb{R})$, then we define and use the following larger class of test functions:

$$
C_{6}:=\left\{h \in H^{K+1}(\mathbb{R}):\|h\|_{L_{\infty}} \leq 1\right\} .
$$

Therefore, the new formulation is

$$
\min _{f \in C_{\bar{f}}} \sup _{h \in C_{6}}\left\{\left\|f-f_{0}\right\|_{L_{2}\left(E_{\mu}\right)}^{2}+2 \lambda \int_{E_{\mu}} f(t) \mathrm{L}^{*} h(t) \mathrm{d} t\right\} .
$$

Using a minimax theorem [6], we change the order of operations to obtain

$$
\sup _{h \in C_{6}} \min _{f \in C_{\bar{f}}}\left\{\left\|f-f_{0}\right\|_{L_{2}\left(E_{\mu}\right)}^{2}+2 \lambda \int_{E_{\mu}} f(t) \mathrm{L}^{*} h(t) \mathrm{d} t\right\} .
$$

The inner minimization problem is solved by orthogonal projection; i.e., the minimizer is

$$
P_{C_{\bar{f}}}\left(f_{0}-\lambda \mathrm{L}^{*} h\right) .
$$

Next, using the equality

$$
\left\|f-\left(f_{0}-\lambda \mathrm{L}^{*} h\right)\right\|_{L_{2}\left(E_{\mu}\right)}^{2}=\|f\|_{L_{2}\left(E_{\mu}\right)}^{2}-2 \int_{E_{\mu}} f(t)\left(f_{0}(t)-\lambda \mathrm{L}^{*} h(t)\right) \mathrm{d} t+\left\|f_{0}-\lambda \mathrm{L}^{*} h\right\|_{L_{2}\left(E_{\mu}\right)}^{2},
$$

we reformulate the objective function as

$$
\left\|f-f_{0}\right\|_{L_{2}\left(E_{\mu}\right)}^{2}+2 \lambda \int_{E_{\mu}} f(t) \mathrm{L}^{*} h(t) \mathrm{d} t=\left\|f-\left(f_{0}-\lambda \mathrm{L}^{*} h\right)\right\|_{L_{2}\left(E_{\mu}\right)}^{2}-\left\|f_{0}-\lambda \mathrm{L}^{*} h\right\|_{L_{2}\left(E_{\mu}\right)}^{2}+\left\|f_{0}\right\|_{L_{2}\left(E_{\mu}\right)}^{2} .
$$

Substituting (4.2) into the objective function, we are left with the following problem:

$$
\sup _{h \in C_{6}}\left\{\left\|P_{C_{\bar{f}}}\left(f_{0}-\lambda \mathrm{L}^{*} h\right)-\left(f_{0}-\lambda \mathrm{L}^{*} h\right)\right\|_{L_{2}\left(E_{\mu}\right)}^{2}-\left\|f_{0}-\lambda \mathrm{L}^{*} h\right\|_{L_{2}\left(E_{\mu}\right)}^{2}\right\}
$$

since we are only concerned with the argument, the term $\left\|f_{0}\right\|_{L_{2}\left(E_{\mu}\right)}^{2}$ is omitted. Multiplying by -1 , we convert this to a minimization problem:

$$
\inf _{h \in C_{6}}\left\{-\left\|P_{C_{\bar{f}}}\left(f_{0}-\lambda \mathrm{L}^{*} h\right)-\left(f_{0}-\lambda \mathrm{L}^{*} h\right)\right\|_{L_{2}\left(E_{\mu}\right)}^{2}+\left\|f_{0}-\lambda \mathrm{L}^{*} h\right\|_{L_{2}\left(E_{\mu}\right)}^{2}\right\} .
$$


If we denote the objective function of (4.3) as $q(h)$, then the formal gradient of $q$ is given by

$$
\nabla q(h)=2 \lambda \mathrm{L}_{C_{\bar{f}}}\left(f_{0}-\lambda \mathrm{L}^{*} h\right) .
$$

We then apply a gradient projection method to approximate the minimizer; for a given value $h_{m-1}$, the next iteration is

$$
h_{m}=P_{C_{6}}\left(h_{m-1}-\frac{2}{\xi} \lambda \mathrm{L} P_{C_{\bar{f}}}\left(f_{0}-\lambda \mathrm{L}^{*} h_{m-1}\right)\right)
$$

where $\xi$ denotes the Lipschitz constant of $\nabla q$.

Suppose we are given the initial guess $f_{0}$, an upper bound $\Xi$ on the Lipschitz constants $\xi$, and the number of iterations $M$. Then Algorithm 1 is used to approximate the projection $P_{\widetilde{C}_{3}} f_{0}$. For improved performance, we implement Algorithm 2 using Nesterov's method [1, 14].
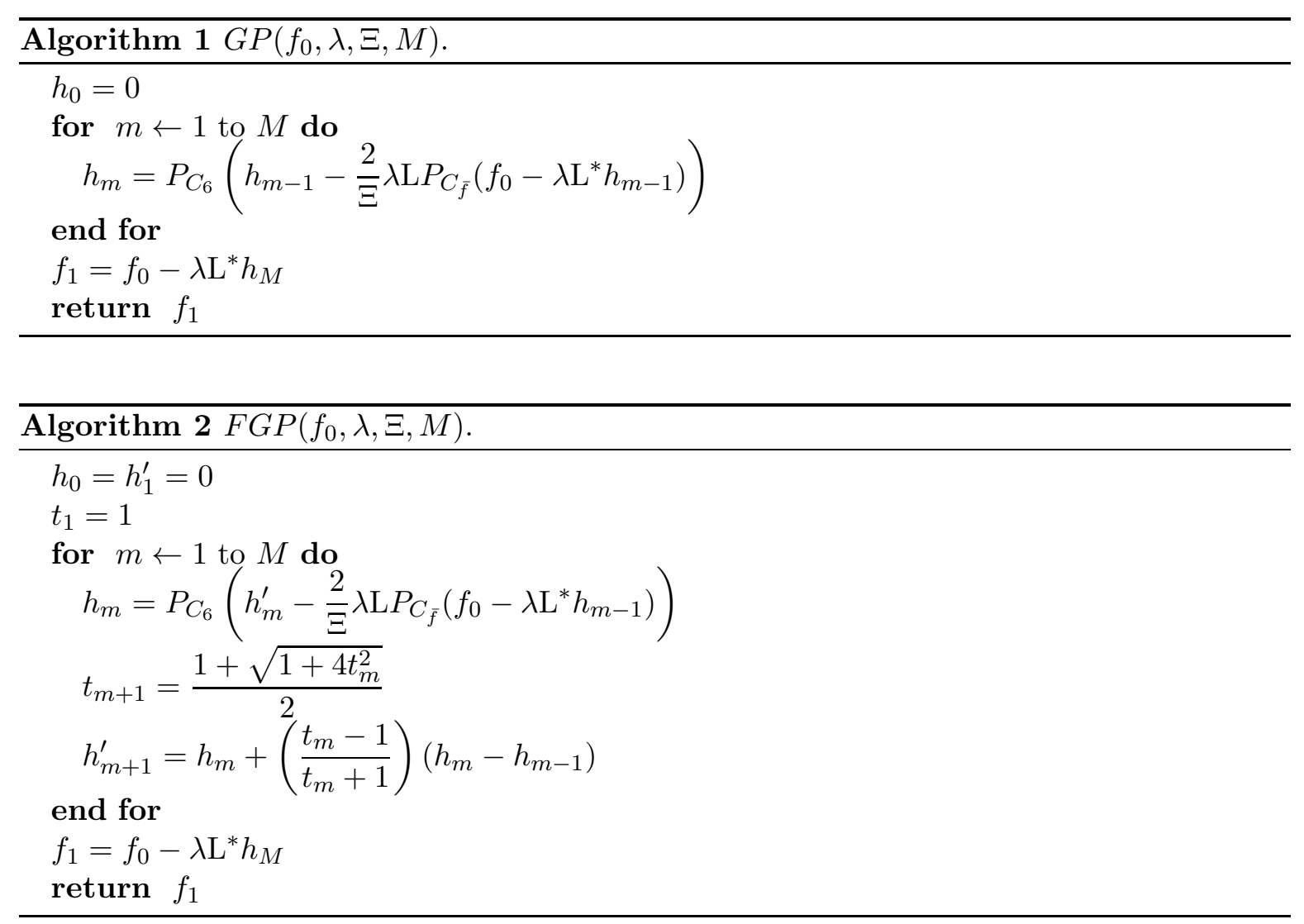

4.3. Simulation results. The following reconstruction experiment uses two-dimensional circular fan-beam projection data that was generated using a ray-driven discrete projector. We use a two-dimensional inner organ phantom (cf. Figure 3 ), which has size $512 \times 512$ with $1 \times 1 \mathrm{~mm}^{2}$ pixel size. This is a virtual human phantom that segments real X-ray computed tomography images. The number of detectors in the array is 390 , with $1 \mathrm{~mm}$ pitch, and the number of views is 1200 for $360^{\circ}$ scanning. The distance from the source to the rotation axis 


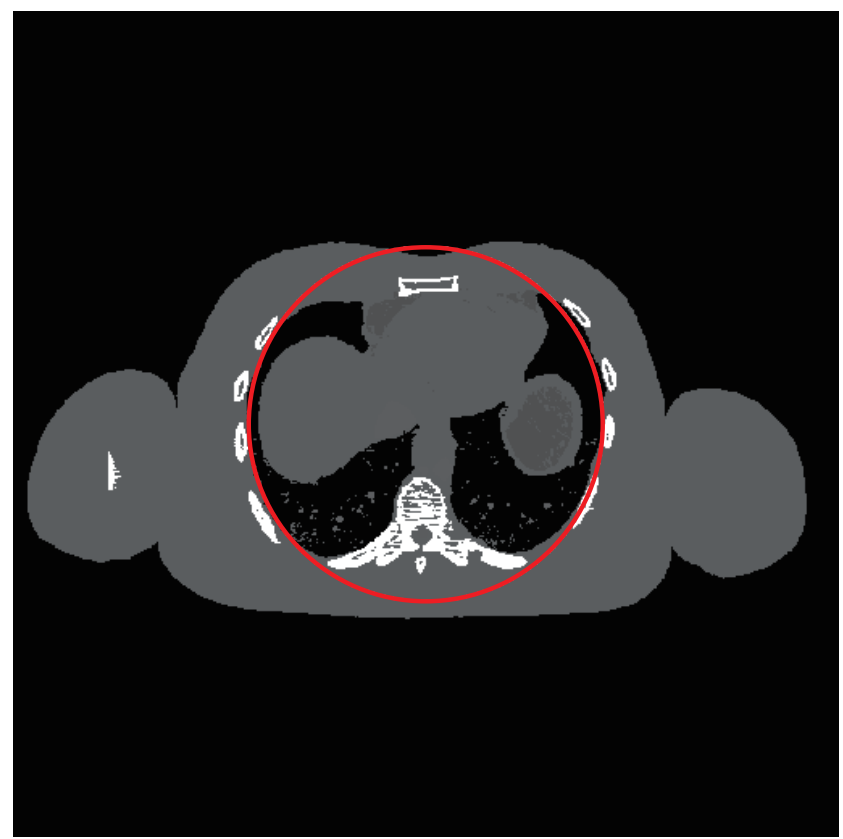

Figure 3. Two-dimensional inner organ phantom. The red circle indicates the FOV.

is $800 \mathrm{~mm}$, and the distance from the source to the detector is $1400 \mathrm{~mm}$. The radius of the ROI is approximately $110 \mathrm{~mm}$, so it does not cover the entire domain.

For implementation, we define a set of chord lines $\left\{l_{n}: n=1, \ldots, N\right\}$ connecting points on the path of the scanner. These chord lines are parallel to the $x_{2}$-axis (cf. Figure 4) and cover the FOV. The line spacing is equivalent to the resolution of the object, and we reconstruct $I$ on these lines. On the intersection between $l_{n}$ and the FOV (the interval $\left(e_{2}, e_{3}\right)$ ), the DBP data is computed from the fan-beam data as in [22]. For this step, we used a pixeldriven backprojector, which is not adjoint to the ray projector. Then we apply the interior tomography algorithm with a generalized TV penalty. With the simulation environment described above and the inner organ phantom in Figure 3, the truncation rate (the length of the support of the line in the FOV $\left(e_{2}, e_{3}\right)$ divided by length of support of the line across the image $\left.\left(e_{1}, e_{4}\right)\right)$ of each chord line ranges from 0 to 0.95 , and the average truncation rate is approximately 0.75 .

Figure 5 shows the reconstructed images for the two-dimensional circular fan-beam simulation. Figure 5(a) displays the phantom only in the ROI. This phantom has complicated structures even though it is piecewise constant, as can be seen in the line profiles of Figure 7. Figure $5(\mathrm{~b})$ is obtained by taking the one-dimensional inverse Hilbert transform of DBP signals. In this case, the overall structure is recovered, but its value is not correct due to truncation. Figures $5(\mathrm{c})$ and $5(\mathrm{~d})$ were created using the proposed interior tomography algorithm with first and second order generalized TV seminorms. In (1.1), $K=1$ and $K=2$ denote first and second order TV, respectively. Comparing the results of Figure 5, we see that the results in (c) and (d) are much better than (b). The first order reconstruction is constant over larger regions, and some of the small structures are lost. However, the second 


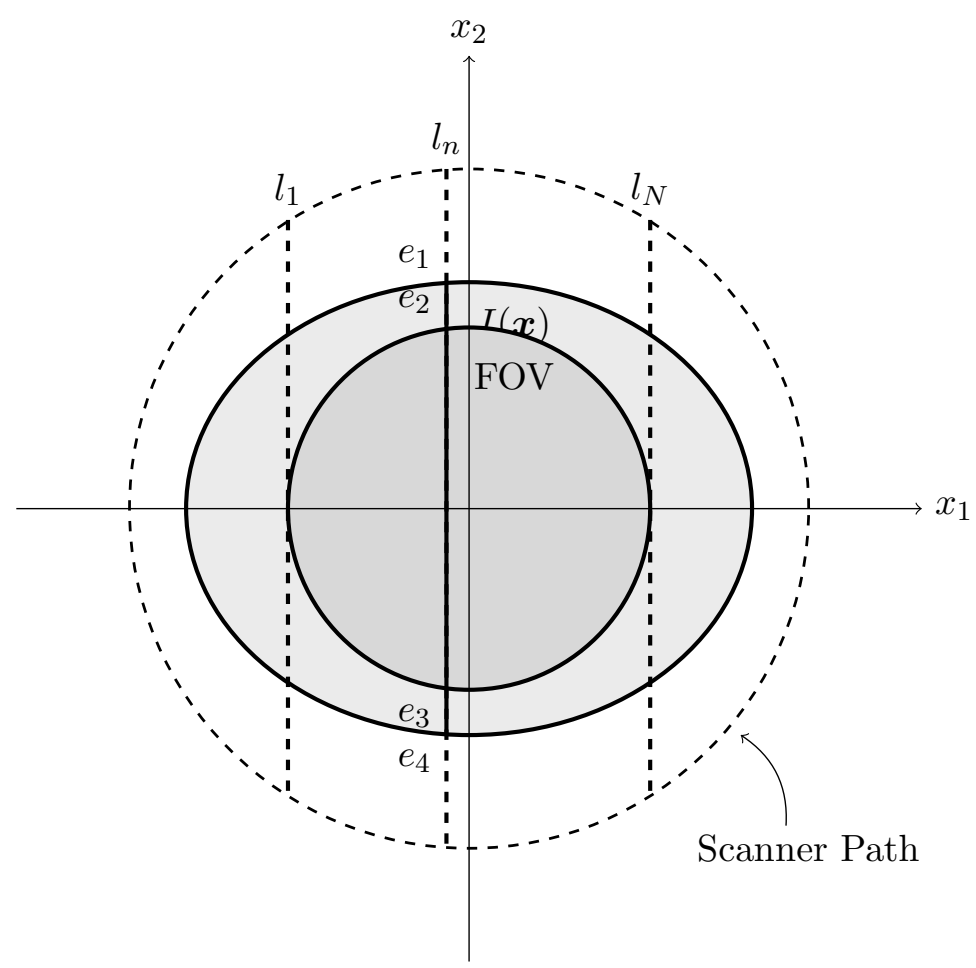

Figure 4. Chord lines for two-dimensional circular fan-beam computed tomography.

order reconstruction seems to more accurately capture these small, fine-detailed structures. Figure 6 contains zoomed regions of the reconstructed images from Figure 5. In Figure 6(c), the reconstruction using the second order TV contains small structures similar to the ground truth even though it is blurred, but in Figure 6(b) all of the structures vanish except the large one.

Figure 7 contains line profiles of the reconstructed images from Figure 5. For each reconstruction result, the values along two chosen chord lines are plotted. In each column, the plots represent different reconstruction techniques on the same chord line. As we have explained, the graphs of the nonregularized method, Figure 7(a), are off by a constant with a roll-off around the edges, even though the shape appears to be correct. In Figures 7(b) and 7(c) the proposed method succeeds in obtaining the correct values in general. Again we see that the second order TV seminorm (Figure 7(c)) produces a better fit to the small structures than the first order TV seminorm (Figure 7(b)).

5. Discussion. In this work, our focus was the mathematical foundation of a tomographic reconstruction algorithm. We have addressed the interior tomography problem for multidimensional images. We proposed a one-dimensional reconstruction procedure that reduces the complexity of the problem, but we are not losing generality. In fact, our approach handles more general images than previous interior tomography methods.

In addition, we have provided some experimental results that support our approach. Some issues that remain to be addressed are the stability of the method and the influence of noise. 


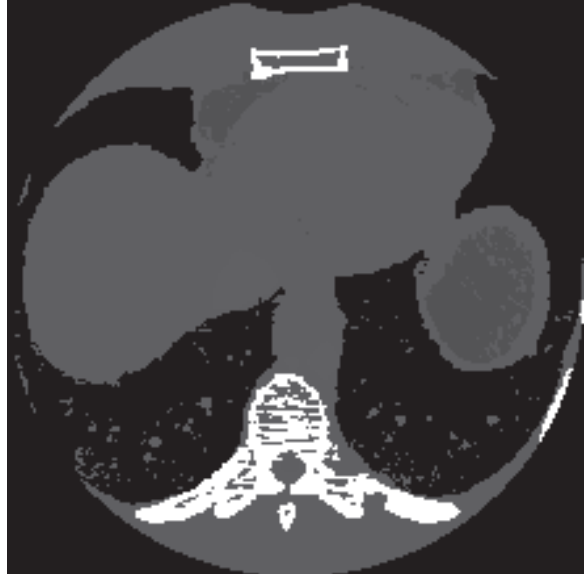

(a) Ground truth

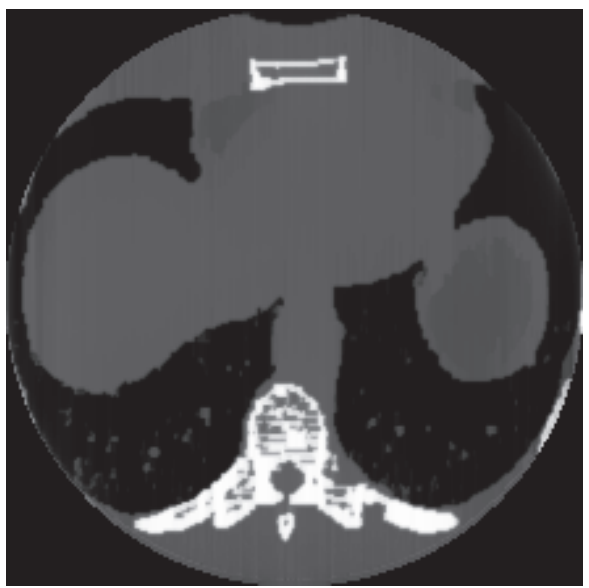

(c) First order TV

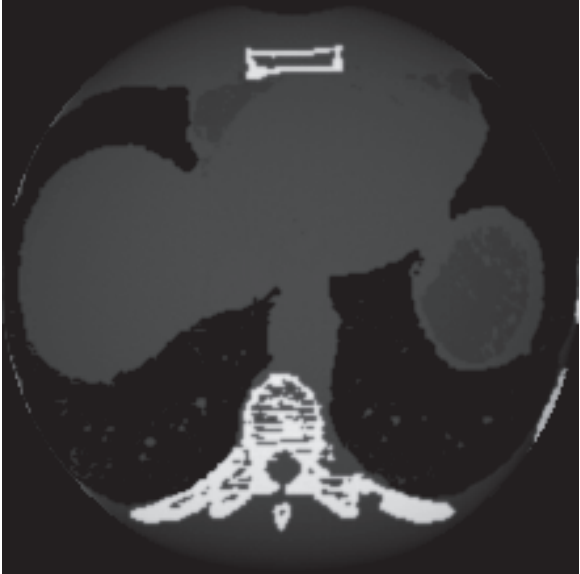

(b) Inverse Hilbert transform

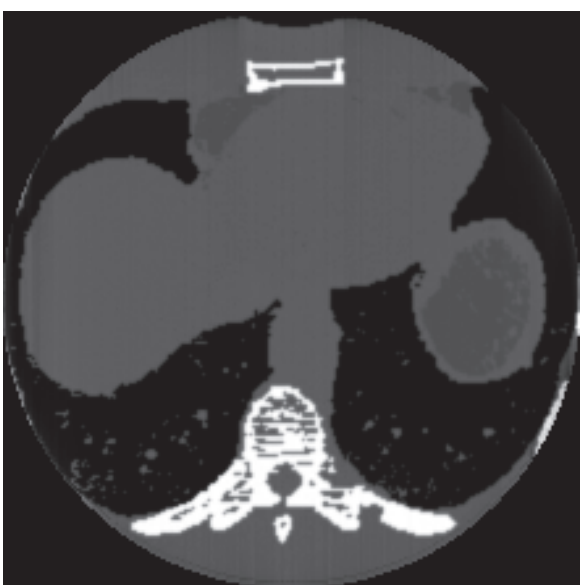

(d) Second order TV

Figure 5. Images reconstructed from simulated two-dimensional circular fan-beam computed tomography data.

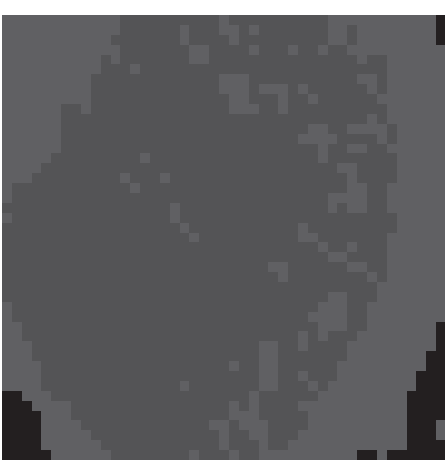

(a) Ground truth

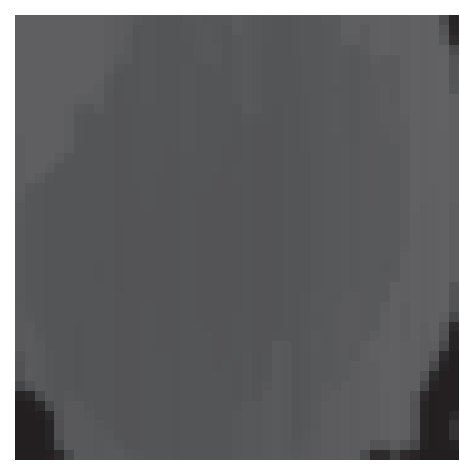

(b) First order TV

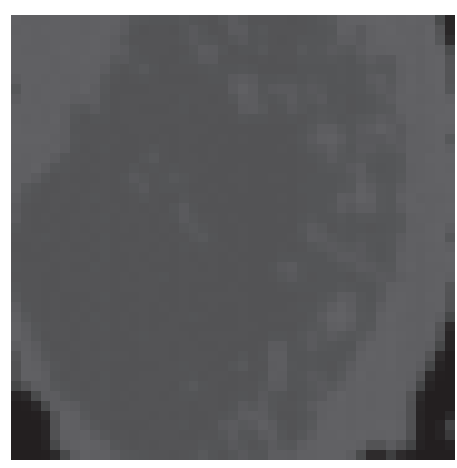

(c) Second order TV

Figure 6. Zoomed reconstruction images from simulated two-dimensional circular fan-beam computed tomography data. 

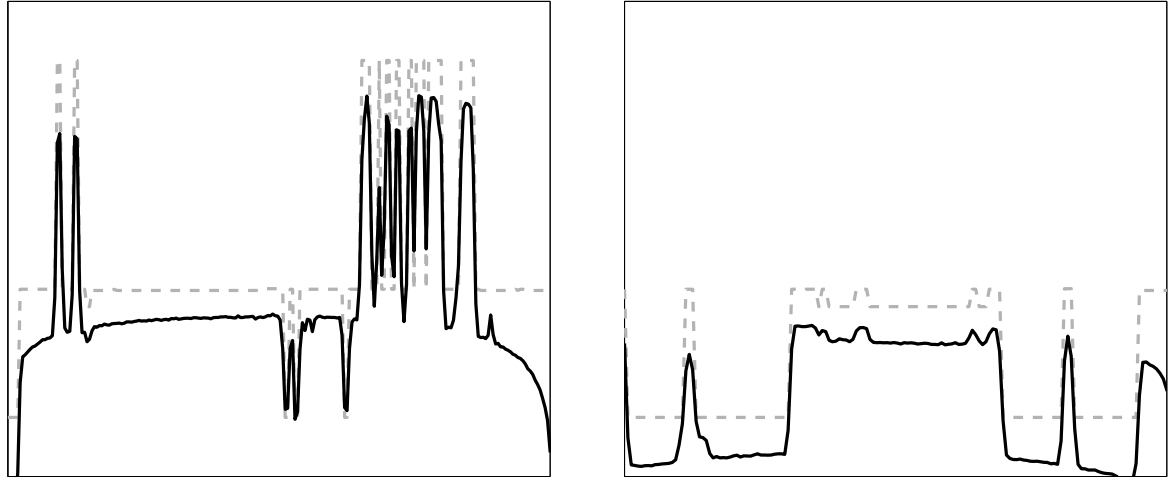

(a) Inverse Hilbert transform
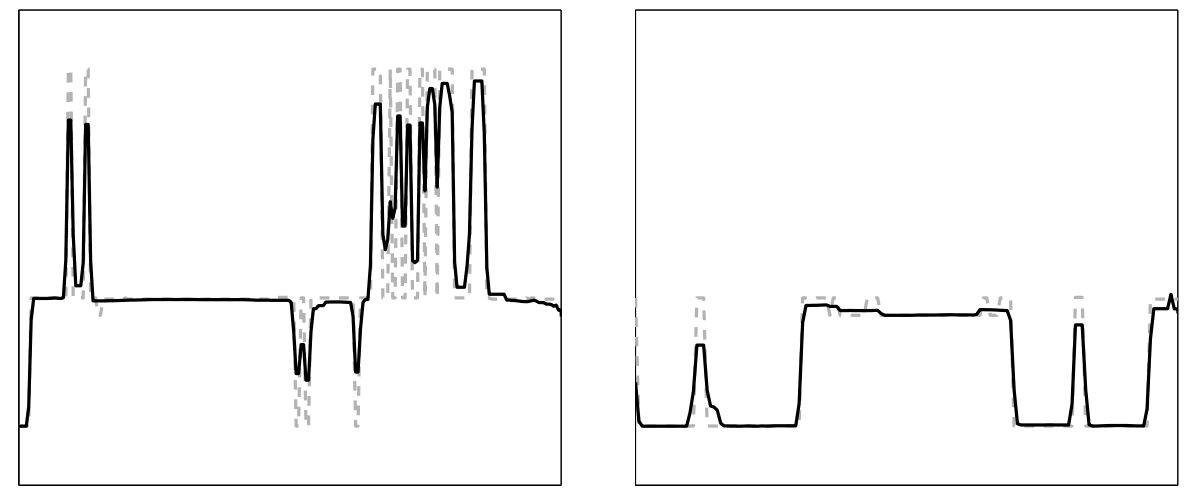

(b) First order TV
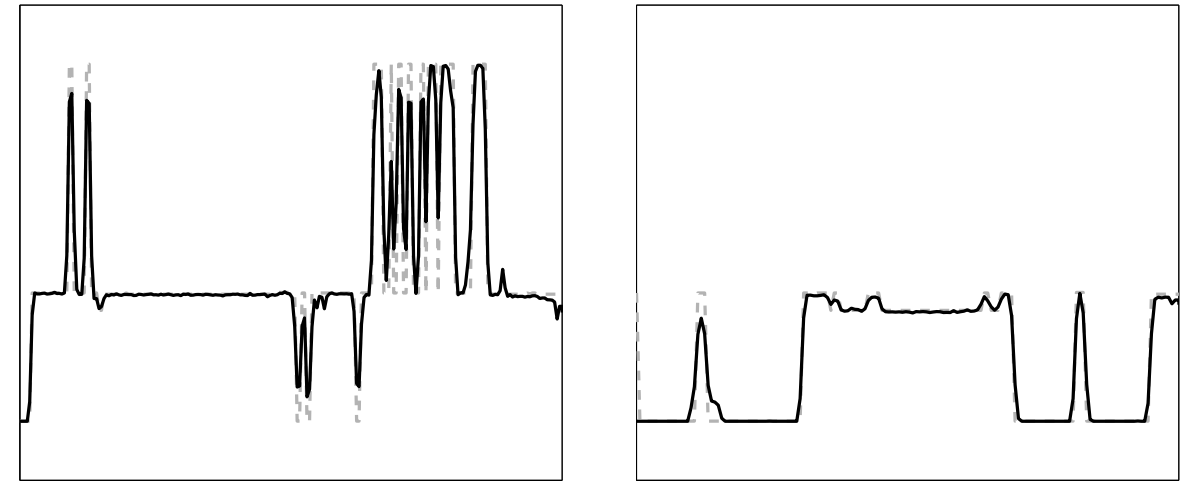

(c) Second order TV

Figure 7. Reconstruction line profiles from simulated two-dimensional circular fan-beam computed tomography data. The solid line represents the reconstruction, while the dashed line is the ground truth.

As we increase the order of the multispline model, we can recover a larger class of functions; however, we expect there to be a trade-off with the stability of the method. Also, in the presence of noise, it may be preferable to choose a lower order model to prevent overfitting

Copyright @ by SIAM. Unauthorized reproduction of this article is prohibited. 
the noisy data. Our early numerical results are promising, and this leads us to move forward with a more detailed evaluation, which will be presented in a future work.

Appendix A. Backprojection operator properties. It is well known that the standard backprojection operator is the adjoint of the Radon operator. Here, we verify that our modified backprojection operator also satisfies a duality property with the Radon operator. Fundamentally, there is no real distinction between these two relationships. Our backprojection operator has a restricted domain, which corresponds to a modified inner product.

Proposition A.1. The backprojection operator $B_{\varphi_{0}}$ is dual to the Radon operator $\mathcal{R}$ in the following sense. For any image $I$ in the $S$ chwartz space $\mathcal{S}\left(\mathbb{R}^{d}\right)$ and any sinogram $J \in \mathcal{S}\left(\mathbb{S}^{d-1} \times\right.$ $\mathbb{R})$, we have

$$
\int_{\mathbb{S}^{d-1} \cap\left\{\phi \cdot \varphi_{0} \geq 0\right\}} \int_{\mathbb{R}} \mathcal{R} I(\boldsymbol{\phi}, s) J(\boldsymbol{\phi}, s) \mathrm{d} s \mathrm{~d} \boldsymbol{\phi}=\int_{\mathbb{R}^{d}} I(\boldsymbol{x}) B_{\boldsymbol{\varphi}_{0}} J(\boldsymbol{x}) \mathrm{d} \boldsymbol{x} .
$$

Proof. For any $\phi \in \mathbb{S}^{d-1}$, we have

$$
\int_{\mathbb{R}} \mathcal{R} I(\boldsymbol{\phi}, s) J(\boldsymbol{\phi}, s) \mathrm{d} s=\int_{\mathbb{R}} \int_{\boldsymbol{\phi}^{\perp}} I(s \boldsymbol{\phi}+\boldsymbol{y}) J(s, \boldsymbol{\phi}) \mathrm{d} \boldsymbol{y} \mathrm{d} s .
$$

Then we change variables, setting $\boldsymbol{x}=s \boldsymbol{\phi}+\boldsymbol{y}$, so

$$
\int_{\mathbb{R}} \mathcal{R} I(s, \boldsymbol{\phi}) J(s, \boldsymbol{\phi}) \mathrm{d} s=\int_{\mathbb{R}^{d}} I(\boldsymbol{x}) J(\boldsymbol{x} \cdot \boldsymbol{\phi}, \boldsymbol{\phi}) \mathrm{d} \boldsymbol{x} .
$$

Now, we integrate both sides with respect to $\phi$ to obtain the result.

$$
\begin{aligned}
\int_{\mathbb{S}^{d-1} \cap\left\{\boldsymbol{\phi} \cdot \boldsymbol{\varphi}_{0} \geq 0\right\}} \int_{\mathbb{R}} \mathcal{R} I(s, \boldsymbol{\phi}) J(s, \boldsymbol{\phi}) \mathrm{d} s \mathrm{~d} \boldsymbol{\phi} & =\int_{\mathbb{R}^{d}} I(\boldsymbol{x}) \int_{\mathbb{S}^{d-1} \cap\left\{\boldsymbol{\phi} \cdot \boldsymbol{\varphi}_{0} \geq 0\right\}} J(\boldsymbol{x} \cdot \boldsymbol{\phi}, \boldsymbol{\phi}) \mathrm{d} \boldsymbol{\phi} \mathrm{d} \boldsymbol{x} \\
& =\int_{\mathbb{R}^{d}} I(\boldsymbol{x}) B_{\boldsymbol{\varphi}_{0}} J(\boldsymbol{x}) \mathrm{d} \boldsymbol{x} .
\end{aligned}
$$

This duality property is used to find the Fourier domain formulation of the backprojection operator, as stated in Lemma 1.3.

Proof of Lemma 1.3. For $I \in \mathcal{S}\left(\mathbb{R}^{d}\right)$, duality implies that

$$
\begin{aligned}
\int_{\mathbb{R}^{d}} I(\boldsymbol{x}) \mathcal{F}\left\{B_{\boldsymbol{\varphi}_{0}} J\right\}(\boldsymbol{x}) \mathrm{d} \boldsymbol{x} & =\int_{\mathbb{R}^{d}} \widehat{I}(\boldsymbol{x}) B_{\boldsymbol{\varphi}_{0}} J(\boldsymbol{x}) \mathrm{d} \boldsymbol{x} \\
& =\int_{\mathbb{S}^{d-1} \cap\left\{\boldsymbol{\phi} \cdot \boldsymbol{\varphi}_{0} \geq 0\right\}} \int_{\mathbb{R}} \mathcal{R} \widehat{I}(\boldsymbol{\phi}, s) J(\boldsymbol{\phi}, s) \mathrm{d} s \mathrm{~d} \boldsymbol{\phi} \\
& \left.=\int_{\mathbb{S}^{d-1} \cap\left\{\boldsymbol{\phi} \cdot \boldsymbol{\varphi}_{0} \geq 0\right\}} \int_{\mathbb{R}^{-1}} \mathcal{F}^{-1} \widehat{\mathcal{I}}(\boldsymbol{\phi}, \cdot)\right\}(\rho) \mathcal{F}\{J(\boldsymbol{\phi}, \cdot)\}(\rho) \mathrm{d} \rho \mathrm{d} \boldsymbol{\phi} .
\end{aligned}
$$

Then we change variables, setting $\boldsymbol{y}=\rho \boldsymbol{\phi}$. Note that if $\rho>0$, then $\boldsymbol{y} \cdot \boldsymbol{\varphi}_{0}>0$ and $\rho=|\boldsymbol{y}|$. When $\rho<0$, we have $\rho=-|\boldsymbol{y}|$. Consequently,

$$
\int_{\mathbb{R}^{d}} I(\boldsymbol{x}) \mathcal{F}\left\{B_{\varphi_{0}} J\right\}(\boldsymbol{x}) \mathrm{d} \boldsymbol{x}=\int_{\mathbb{R}^{d}} I(\boldsymbol{y}) \mathcal{F}\left\{J\left(\operatorname{sgn}\left(\boldsymbol{y} \cdot \boldsymbol{\varphi}_{0}\right) \frac{\boldsymbol{y}}{|\boldsymbol{y}|}, \cdot\right)\right\}\left(\operatorname{sgn}\left(\boldsymbol{y} \cdot \boldsymbol{\varphi}_{0}\right)\right)|\boldsymbol{y}|^{1-d} \mathrm{~d} \boldsymbol{y} .
$$


Acknowledgment. We thank the reviewers for their helpful comments.

\section{REFERENCES}

[1] A. Beck And M. Teboulle, Fast gradient-based algorithms for constrained total variation image denoising and deblurring problems, IEEE Trans. Image Process., 18 (2009), pp. 2419-2434.

[2] R. P. BoAs, Invitation to Complex Analysis, 2nd ed., MAA Textbooks, Mathematical Association of America, Washington, DC, 2010; revised by H. P. Boas.

[3] M. Courdurier, F. Noo, M. Defrise, And H. Kudo, Solving the interior problem of computed tomography using a priori knowledge, Inverse Problems, 24 (2008), 065001.

[4] M. Defrise, F. Noo, R. Clackdoyle, and H. Kudo, Truncated Hilbert transform and image reconstruction from limited tomographic data, Inverse Problems, 22 (2006), pp. 1037-1053.

[5] J. Duchon, Splines minimizing rotation-invariant semi-norms in Sobolev spaces, in Constructive Theory of Functions of Several Variables, W. Schempp and K. Zeller, eds., Lecture Notes in Math. 571, Springer, Berlin, Heidelberg, 1977, pp. 85-100.

[6] K. FAn, Minimax theorems, Proc. Natl. Acad. Sci. USA, 39 (1953), pp. 42-47.

[7] I. M. GeL'FAND AND M. I. Graev, The Crofton function and inversion formulas in real integral geometry, Funktsional. Anal. i Prilozhen., 25 (1991), pp. 1-6.

[8] L. Grafakos, Classical Fourier Analysis, 2nd ed., Grad. Texts in Math. 249, Springer, New York, 2008.

[9] F. W. King, Hilbert Transforms, Volume 1, Encyclopedia Math. Appl. 124, Cambridge University Press, Cambridge, UK, 2009.

[10] F. W. King, Hilbert Transforms, Volume 2, Encyclopedia Math. Appl. 125, Cambridge University Press, Cambridge, UK, 2009.

[11] S. LAng, Complex Analysis, 4th ed., Grad. Texts in Math. 103, Springer-Verlag, New York, 1999.

[12] M. Lee, J. P. WARd, M. Unser, AND J. C. Ye, Multiscale interior tomography using $1 D$ generalized total variation, in Proceedings of the Third International Conference on Image Formation in X-Ray Computed Tomography, 2014, pp. 347-350.

[13] F. NATTERER, The Mathematics of Computerized Tomography, B. G. Teubner, Stuttgart, 1986.

[14] Y. E. Nesterov, A method for solving the convex programming problem with convergence rate o $\left(1 / k^{2}\right)$, Dokl. Akad. Nauk SSSR, 269 (1983), pp. 543-547 (in Russian).

[15] F. Noo, R. Clackdoyle, and J. D. Pack, A two-step Hilbert transform method for $2 D$ image reconstruction, Phys. Med. Biol., 49 (2004), pp. 3903-3923.

[16] E. M. Stein, Singular Integrals and Differentiability Properties of Functions, Princeton Math. Ser. 30, Princeton University Press, Princeton, NJ, 1970.

[17] G. Wang And H. Yu, The meaning of interior tomography, Phys. Med. Biol., 58 (2013), pp. R161-R186.

[18] J. YANG, H. YU, M. JIANG, AND G. WANG, High-order total variation minimization for interior tomography, Inverse Problems, 26 (2010), 035013.

[19] Y. Ye, H. Yu, AND G. WAng, Gel' fand-Graevs reconstruction formula in the $3 D$ real space, Med. Phys., 38 (2011), pp. S69-S75.

[20] H. Yu And G. WAng, Compressed sensing based interior tomography, Phys. Med. Biol., 54 (2009), pp. 2791-2805.

[21] Y. Zou AND X. PAN, Exact image reconstruction on PI-lines from minimum data in helical cone-beam CT, Phys. Med. Biol., 49 (2004), pp. 941-959.

[22] Y. Zou AND X. PAN, Image reconstruction on PI-lines by use of filtered backprojection in helical cone-beam CT, Phys. Med. Biol., 49 (2004), pp. 2717-2731.

[23] Y. Zou, X. PAN, AND E. Y. SIDKY, Image reconstruction in regions-of-interest from truncated projections in a reduced fan-beam scan, Phys. Med. Biol., 50 (2005), pp. 13-27.

Copyright $\odot$ by SIAM. Unauthorized reproduction of this article is prohibited. 